\title{
A Study on the Change of the Fe La Mass Absorption Coefficients and Fluorescence Yields in Iron Silicide Samples by EPMA
}

\author{
Aurélien Moy ${ }^{1}$ and John Fournelle ${ }^{1}$ \\ 1. Department of Geoscience, University of Wisconsin, Madison, USA.
}

The study of natural iron silicides is of importance because the extreme reducing environment required for their formation can give insight into the mechanism that created them. The presence of iron silicides has been reported in a variety of environments, from lightning-induced fulgarites to micro-meteoritic impact-formed lunar regolith and stardust samples. Natural iron silicide phases can be relatively small (few $\mu \mathrm{m}$ ) and their quantification by EPMA can be difficult [1]. Indeed, despite the recent introduction of the field emission gun (FEG), which allows sub-micron resolution imaging with a very small electron beam, the X-ray lateral spatial analytical resolution remains too large at traditional accelerating voltages (e.g., $15 \mathrm{kV}$ ). To improve analytical resolution, the accelerating voltage can be decreased to $5-7 \mathrm{kV}$, reducing the typical size of the electron interaction volume from $\sim 1-2 \mu \mathrm{m}$ to $\sim 0.2-0.7 \mu \mathrm{m}$ (depending upon composition). However, by reducing the accelerating voltage, the $\mathrm{K} \alpha$ line of some elements of the periodic table cannot be excited. The L lines must then be used for these elements, but these L X-ray lines are not well known for the first-row transition metals and complex chemical and physical effects can occur. Indeed, the L $\alpha$ X-ray line of those elements involves the partially filled $3 \mathrm{~d}$ electron shell which is subject to strong bonding effects with the electrons of the neighboring atoms. As a result, the outer electronic shell energies are modified by these bonding effects and then are the associated transition probabilities. The different atomic parameter values (mass absorption coefficient (MAC), fluorescence yield, Coster-Kronig factor, ...) not only depend of the studied element but also of the composition and nature of the material, both of the unknown and of the standard used. This led Gopon et al. [1] to successfully demonstrate the utility of the Fe Ll line. While this is a useful alternative, there remains the issue of the low intensities of Fe Ll X-rays. This has led us to a further examination of the Fe L $\alpha$ line (with its high count rate) for quantitative EPMA.

The present work focuses on the study of the MAC of the Fe L $\alpha$ X-ray line by the element Fe and of the fluorescence yield of the Fe L $\alpha$ line, in 6 bulk synthetic iron silicide compounds and in one bulk Fe standard. Their compositions were verified by EPMA (CAMECA SXFive FE) at 10, 15, 20 and $25 \mathrm{kV}$ (at $20 \mathrm{nA}$ ). The element Fe was analyzed using the Ka X-ray line with LIF crystals, and Si was analyzed using the K $\alpha$ X-ray line with LPET crystals. Ten points were acquired on each sample at each accelerating voltage. The measurements were also performed on pure Fe and Si standards. The samples were Pt-coated to ensure electrical conductivity. The coating thickness was determined by measuring the Pt M $\alpha$ k-ratio on the samples and a pure Pt standard, at 7 and $15 \mathrm{kV}$, and analyzed with the software STRATAGem, indicating a Pt coating thickness of $5.5 \AA$. The measured compositions of Fe in the iron silicides, using the PAP model [2], vary from 46 to $89 \mathrm{wt} \%$.

Next, the Fe L $\alpha$ and Si K $\alpha$ X-ray lines were measured at 2.5, 3, 5, 7, 10, 15, 20, 25 and 30 kV (@90 nA) using a TAP and a LTAP crystals for the Fe and 2 LPET crystals for the Si. The traditional method of measuring the X-ray intensity on the maximum of the peak and on each side to remove the background is not valid here for the Fe L $\alpha$ line because of the strong auto-absorption on the high energy side of the line. The magnitude and shape of this absorption depends on both the accelerating voltage used and the composition of the sample, because of different bonding effects. The measured intensities using this method are therefore not comparable between the standard and the iron silicide compound. A more proper way to measure the intensity is to record a spectrum of the L $\alpha$ line, deconvolute it, and to integrate it to 
determine the total amount of X-ray detected. For each sample and each accelerating voltage, three spectra were recorded and averaged, and then fitted with a set of pseudo-Voigt functions to determine the $\mathrm{X}$-ray intensities of the deconvoluted components.

The MACs were obtained by non-linear fitting of the Fe La X-ray intensities in relation to the accelerating voltage using theoretical intensities calculated with the PAP model [2] with the MAC of the $\mathrm{Fe} L \alpha$ line in the specific sample set as a free fitting parameter. A decrease of the curve at low accelerating voltage (from 2.5 to $5 \mathrm{kV}$ ) is seen on all the iron silicide samples, indicating the presence of a strong absorption of the $\mathrm{Fe} \mathrm{L} \alpha$ and $\mathrm{Si} \mathrm{K} \alpha \mathrm{X}$-ray lines by a light element, probably $\mathrm{O}$ due to natural oxidation of the samples. Qualitative EPMA measurements confirm the presence of oxygen on the samples. Furthermore, this decrease is only reproduced by the calculations if a layer of $\mathrm{Pt}$ and/or a layer of oxidized iron silicide is taken into account. The obtained values of the MAC vary greatly for the low Fe concentration samples compared to the high Fe ones as shown on Figure 1. The variation of the MAC does not seem to follow a linear trend as indicated by the two values obtained for the low $\mathrm{Fe}$ concentration samples. This may be due to a change in the crystal structure of the compounds, affecting the bonding of the $\mathrm{Fe}$ atoms. Further investigations of this are currently underway. An attempt to synthesize additional samples with very low and intermediate concentrations of $\mathrm{Fe}$ is also in progress, to be used to determine if a relationship between Fe concentration, crystal structure and MAC value exists.

As demonstrated by Llovet et al. in a recent paper [3], the fluorescence yield value is also greatly changed by the composition of the sample for the transition metals. Therefore, the PAP theoretically calculated kratios and measured k-ratios differ by a scaling factor. For a given iron silicide sample, by enhancing the fluorescence yield by this scaling factor, the calculated k-ratios are in good agreement with the experimental data. Figure 2 shows the fluorescence enhancement yield factor versus the Fe concentration. With a very good agreement, the curve follows a linear trend.

This linear evolution can then be used in the matrix correction algorithm to calculate the correct fluorescence value and, if a relationship is found for the MACs, will allow the prediction of more accurate quantification results using the $\mathrm{Fe} \mathrm{L} \alpha \mathrm{X}$-ray line.

\section{References:}

[1] P Gopon et al, Microsc. Microanal. 19 (2013), p. 1698.

[2] J L Pouchou and F Pichoir, "Quantitative analysis of homogeneous or stratified microvolumes applying the model PAP", ed. K F J Heinrich and D E Newbury, (Plenum Press) p. 31.

[3] X Llovet et al, Microsc. Microanal. 22 (2016), p. 1233.

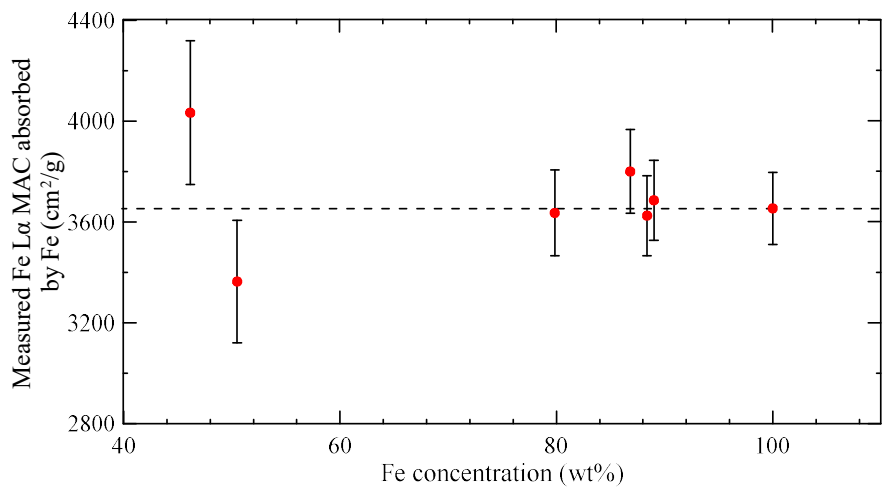

Figure 1. Measured $\mathrm{Fe} \mathrm{L} \alpha \mathrm{MACs}$ (absorbed by $\mathrm{Fe}$ ) displayed as a function of the Fe concentration.

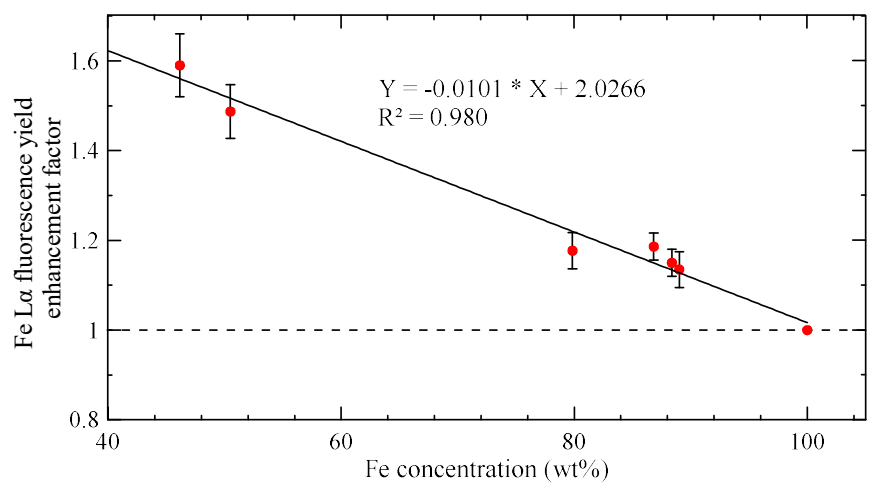

Figure 2. Fluorescence yield enhancement factor measured as a function of the Fe concentration. 